\title{
Captures
}

Figures, théories et pratiques de l'imaginaire

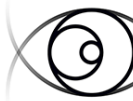

C A P T U R E S Figures, théories et pratiques de limaginaire revue interdisciplinaire

\section{Dieu a planté son compas dans la Seine}

"Paris " épinglé par Vigny

\section{Sébastien Roldan}

Volume 2, Number 2, 2017

Imaginaire de la ligne

URI: https://id.erudit.org/iderudit/1059773ar

DOI: https://doi.org/10.7202/1059773ar

See table of contents

Publisher(s)

Figura, Centre de recherche sur le texte et l'imaginaire

ISSN

2371-1930 (digital)

Explore this journal

Cite this article

Roldan, S. (2017). Dieu a planté son compas dans la Seine : « Paris » épinglé par Vigny. Captures, 2(2). https://doi.org/10.7202/1059773ar
Article abstract

What is it that one sees at night, in 1831, atop a high tower rising from the heart of Paris? Alfred de Vigny's poetic answer to this is fascinating: ridges, angles, rims, summits, needles, lit fires, lightning, but also shadows, vague shapes, wide chasms... Fine lines and large ensembles are opposed and combined to make the city into a compass of titanic proportions, directing the onward march of a world that has lost its sense of direction, yet is going ever faster. This article attempts to shed some light into the fray.
Tous droits réservés @ Cébastien Roldan, 2017

\section{(c) (i) $\Theta$}

This document is protected by copyright law. Use of the services of Erudit (including reproduction) is subject to its terms and conditions, which can be viewed online.

https://apropos.erudit.org/en/users/policy-on-use/ 
Version enrichie de cet article : http://revuecaptures.org/node/927

\section{Dieu a planté son compas dans la Seine}

\section{« Paris » épinglé par Vigny}

\section{Sébastien Roldan}

Résumé :

Que voit-on en 1831, la nuit, du haut d'une tour s'élevant au beau milieu de Paris? La réponse qu'offre Alfred de Vigny est fascinante : arêtes, angles, cimes, aiguilles, feux, éclairs, zébrures, mais aussi ombres, masses informes, gouffres... Délinéaments fins et vastes ensembles courbes s'affrontent et s'unissent pour transfigurer la ville en titanesque compas chargé d'orienter la marche de plus en plus rapide d'un monde en perte de repères. C'est à les démêler que s'applique cet article.

What is it that one sees at night, in 1831, atop a high tower rising from the heart of Paris? Alfred de Vigny's poetic answer to this is fascinating: ridges, angles, rims, summits, needles, lit fires, lightning, but also shadows, vague shapes, wide chasms... Fine lines and large ensembles are opposed and combined to make the city into a compass of titanic proportions, directing the onward march of a world that has lost its sense of direction, yet is going ever faster. This article attempts to shed some light into the fray.

En avril 1831, Alfred de Vigny publie un long poème intitulé «Paris », qui compte 258 vers, tous alexandrins. L'œuvre, simultanément parue dans le Courrier de l'Europe et sous forme de petite plaquette de 27 pages (Jarry, 1973: 289), porte la marque des grands remous politiques agitant cette période particulièrement houleuse pour une grande ville que Vigny envisage déjà en Capitale du siècle, en cœur palpitant de l'ère moderne. C'est un « centre de vie » (Vigny, 1950 [1859]: 111, v. 73) entraînant à sa suite, avec le despotisme d'un « impassible Roi » $(110, v$. 50), tous les environs, à la manière d'une titanesque roue dont les milliers de rayons rejoindraient les contrées les plus proches et les plus lointaines pour les attacher à son train. «Paris, l'axe immortel, Paris, l'axe du monde » (110, v. 51) est pressenti par Vigny, vingt ans avant Charles Baudelaire, comme le noyau fertile des bouleversements en cours 1 . L'image poétique mise en avant est celle d'une ville agissant à la fois en moteur et en moyeu des temps nouveaux.

Il faut nous remettre dans le contexte de l'époque. Si la France sous la Restauration était un monde encore préindustriel, aux modes de production très artisanaux, différent en cela de l'Angleterre $\underline{2}$, les débuts de la Monarchie de Juillet laissaient présager le formidable impact qu'allait avoir sur le travail l'avènement de la grande machine et de l'industrie (hauts-fourneaux, chaudières à vapeur). Les bouleversements ne sont pas moindres dans le monde politique, comme le rappelle Paul Bénichou : «l'échec de la Restauration manifestait le caractère irréversible des changements qui s'étaient produits en France, et posait le problème de l'avenir. » (117.) Soulignons de plus que la publication du poème par Vigny, en avril 1831, coïncide avec 
l'adoption de la nouvelle loi électorale qui, dans la foulée des Trois Glorieuses, étendait, modestement, il est vrai, le droit de vote 3 .

Le régime en place, certes plus laïque, tolérant et progressiste que le précédent, n'avait pas encore neuf mois; et il accouchait, prématurément peut-être, de réformes encourageantes quoique insatisfaisantes. Les débats faisaient rage; les écoles de pensée s'affrontaient emplies d'espoir, dans un bouillonnement d'idées qui allait conduire les Républicains et les Légitimistes à multiplier les émeutes, les complots, les tentatives d'insurrection. Au plan littéraire, le Paris médiéval est à la mode chez les romanciers 4 , mais la ville moderne de même que la geste de la Révolution de 1830 font aussi l'objet de nombreuses évocations, en tous genres. Vigny lui-même s'apprête à revenir sur le sujet dans Stello, dont les premiers épisodes commencent à paraître en préoriginale quelques mois seulement après « Paris $\underline{5}$ », si bien qu'on a pu envisager l'urbanité décrite dans le roman comme indissociable de son antécédent versifié (Petroni, 1990: 21). II reste que la poésie de Paris et des Trois-Jours de juillet ne s'est pas encore, à ce stade précoce, figée en formes et figures communément partagées; or, le poème de Vigny constitue un jalon primordial (Citron, 1961:278) au sein du processus de cristallisation des images qui, bientôt reconnaissables et réutilisables à l'infini, composeront le mythe de Paris $\underline{6}$.

À tant d'égards, donc, c'est une heure d'instabilité où l'univers des possibles paraît grand ouvert. Pour en rendre compte, Vigny dans son poème innove en proposant une vue nocturne de la grande ville $\underline{7}$, où l'obscurité nimbe de mystère les forces en jeu et exige un effort accru de la part des témoins du tumulte, lesquels peinent à démêler les grandes lignes, au sens propre comme au figuré, du spectacle de la modernité qui s'offre à eux. La réflexion que propose le présent article vise à creuser l'opposition entre courbures et arêtes que donne à lire Vigny dans « Paris ». Bien entendu, la vision de la ville qui s'y trouve est justement celle d'un lieu moderne, polymorphe, où tous les contraires, où toutes les possibilités se rencontrent, cohabitent, concourent, s'imbriquent et s'altèrent entre eux, créant sans cesse du mouvement, de l'inédit à foison. Ni pour Vigny ni pour nous il ne s'agit de déterminer laquelle, de l'arête acérée ou de la surface courbe, l'emporte à Paris en 1830. La ville dans ce poème est, comme le souligne Paul Bénichou, " énigme et promesse, atelier fabuleux des nouvelles destinées de l'homme » (141). Aussi, notre enquête interroge plutôt le rôle symbolique qui est dévolu à la ligne précise et à son opposée, la courbe diffuse, au sein du système de représentation mis en œuvre par Vigny. Car les lignes de « Paris » sont peut-être brisées, plus souvent qu'autrement hachurées, jetées çà et là, pêle-mêle à travers l'espace urbain décrit par le poète, sujettes à une formidable violence destructrice et agentes de celle-ci, elles n'en dessinent pas moins, globalement, une géométrie significative qui s'inscrit comme porteuse de considérations historiques et poétiques.

Afin d'y voir plus clair, il nous faudra d'abord rappeler le virage esthétique que la sensibilité romantique, dont Vigny est partie prenante, impose au sein des beaux-arts de l'époque, pour ensuite en constater les 
répercussions sur l'image de la réalité urbaine réel qui se dégage de « Paris ». Car la vision de l'actualité y est troublée en ce contexte de changement accéléré des débuts de l'ère industrielle. Aussi en vient-on à interroger Paris directement — sans pour autant obtenir de réponse satisfaisante, ce qui relance l'inquiétude devant un monde opaque et frénétique, apparemment emballé dans sa course, allant devant lui malgré tout, sans but défini. Finalement, c'est en sondant le passé lointain, d'après les indices glanés au fil de ce parcours à travers les âges de la grande Cité, que nous parvenons à conférer une profondeur historique et une épaisseur sémantique à ce geste violent qui, dans les premiers vers du poème de Vigny, nous arrête : celui de Dieu plongeant un compas au milieu des eaux de la Seine.

\section{Opposer pour mieux voir}

Trois courants de pensée majeurs de l'époque se rencontrent dans ce « Paris » qu'esquisse Vigny : le christianisme social rénové de Lamennais, le libéralisme parlementaire de Benjamin Constant et le nivellement égalitariste des saint-simoniens. Pour les présenter, Vigny donne à son poème la forme d'un dialogue entre deux personnages. L'un est désigné par quatre fois comme « Voyageur » (Vigny, 1950 [1831]: 109, v. 11; 111, v. 73; 114, v. 202; 115, v. 255), l'autre demeure anonyme tout au long : appelons-le « Poète ». Après avoir assez longuement évoqué le « triple labeur » $(115$, v. 245) de Lamennais, de Constant et de Saint-Simon, le Poète soudain s'écrie :

- Ainsi, tout est osé! Tu vois? pas de statue

D'homme, de roi, de Dieu, qui ne soit abattue,

Mutilée à la pierre et rayée au couteau,

Démembrée à la hache et broyée au marteau! (112, v. 143-146.)

On aura peut-être remarqué l'imagerie déployée dans ces quatre vers. La violence faite au passé et à ses icônes est associée aux meurtrissures induites par la ligne. Les arêtes acérées du couteau, de la hache et de la pierre rayent, fendent, sectionnent les volumes pleins, les surfaces unies. Corollairement, tout plan lisse se trouve hérissé d'entailles, de zébrures, qui sont autant de nouvelles arêtes. Le travail de destruction opère une multitude de coupures au sein des continuités; aux rondeurs pleines du bras de telle statue, au galbe du torse de telle autre, l'œuvre de démolition substitue d'innombrables lignes qui, plus courtes que celles prévalant avant le saccage, hachurent la matière en menus grains rocailleux. Surgit le spectacle de ces grandes figures de marbre ou de bronze écroulées au pied de leurs piédestaux, jonchant le parterre dans un entassement de pièces désarticulées. Même le travail du marteau, masse pleine s'il en est une, produit ici quantité d'arêtes nouvelles par le morcellement : au lieu de clouer, d'écraser ou de trouer la surface, il broie les volumes, c'està-dire qu'il égrène la matière, pulvérise les conglomérats. 
Pourtant, à lire les quelques vers qui viennent immédiatement à la suite, nous constatons que les segments ainsi générés, parcourus d'arêtes qui sont autant de nouvelles lignes courtes, sont aussitôt refondus en entités pleines, quoique indéfinies. Par l'indistinct, la matière se voit restituer ses continuités et son unité brièvement rompues :

Or ou plomb, tout métal est plongé dans la braise

Et jeté pour refondre en l'ardente fournaise.

Tout brûle, craque, fume et coule; tout cela

Se tord, s'unit, se fend, tombe là, sort de là;

Cela siffle et murmure ou gémit; cela crie,

Cela chante, cela sonne, se parle et prie;

Cela reluit, cela flambe et glisse dans l'air,

Éclate en pluie ardente ou serpente en éclair. (112-113, v. 147-154.)

Sur ces huit vers, la scansion des « tout » et des « cela », ou pour le dire autrement l'évocation d'ensembles imprécis et de flous indéterminés, s'accompagne d'un registre d'images renvoyant à la courbe retorse qui " serpente ", aux vrilles de la vapeur qui s'envole ou à la sphéricité des gouttes d'une " pluie ardente ». Cette dernière formule dit bien que l'identité de chaque tison zébrant le ciel nocturne se perd parmi la multitude de ses semblables.

La courbure et la sphéricité, dans ce poème, sont associées à des unités plurielles vaguement définies ou à des instances difficilement identifiables, impossibles à délimiter ou à désigner avec précision, tant elles sont diffuses, multiples ou étendues. Or, lorsque, au sein de cette même strophe, quelques vers plus bas, reparaît la locution « tout cela » $(113$, v. 158), il devient clair que dans le «Paris » de Vigny l'univers des touts est orienté vers l'avenir et vécu sur le mode de l'euphorie esthétique :

Paris! principe et fin! Paris! ombre et flambeau!

— Je ne sais si c'est mal, tout cela; mais c'est beau!

Mais c'est grand! mais on sent jusqu'au fond de son âme

Qu'un monde tout nouveau se forge à cette flamme [...]. (113, v. 157-160.)

La ligne, en revanche, paraît quant à elle appartenir au domaine des menaces connues, des violences nommables, des entités familières et bornées, liées à quelque passé avéré ou à quelque présent figurable (quoique en voie de disparaître).

L'ensemble du poème de Vigny résonne avec le passage, à la période romantique, d'une esthétique picturale éprise de délinéaments et d'ordonnancements vers une conception du beau davantage liée à la couleur, au 
mouvement, au débraillé luxuriant, au syncrétisme fantaisiste. En peinture, la fin de la Restauration et le début de la Monarchie de juillet marquent, on le sait, la transition entre néoclassicisme et romantisme. Le souci premier du dessin, l'attention à l'effet expressif des lignes, la prédilection pour la netteté, pour la noblesse du trait et les contours bien définis, pour la beauté et l'ordre antiques, pour les sujets « élevés » représentés par David et son école, par Gros, par Ingres, toujours à débusquer l'éloquente contorsion des formes, cèdent progressivement le pas aux sujets modernes ou orientalisants, dans un engouement naissant pour les consonances de la composition, pour le touffu, pour le faisandé maladif, que privilégient Géricault ou Delacroix. Une remarque du Journal d'un poëte(1867) datée de 1834 atteste que Vigny eut tôt conscience de ces pôles artistiques opposés : «Ingres est trop pur de dessin, [...] Delacroix trop coloriste » (Vigny, 1867: 92). Le déplacement du goût de l'un vers l'autre de ces peintres fut notoirement enregistré par Baudelaire critique d'art en 1845-46, après avoir été problématisé par Balzac dès 1837요. Le futur auteur du Peintre de la vie moderne (1863) oppose « les finesses laborieuses à l'harmonie d'ensemble » (1999 [1845]: 59) et tranche en faveur de la « couleur, large, simple, abondante en masses harmoniques » (1999 [1846]: 171) prodiguée par celui qu'il considère comme « le chef de l'école moderne 9 » (1999 [1846]: 152). Coloristes contre dessinateurs $\frac{10}{}$, querelle apparemment indissoluble. Le siècle, fédéré presque d'un bout à l'autre par l'opposition entre ces deux pôles, s'est plu à la résumer comme suit : « le classicisme est la ligne, le romantisme la couleur11 » (Gaucher, 1873: 971).

En phase avec l'esprit romantique, la valeur positive qu'assigne Vigny au diffus, au vague, à la multiplicité indistincte qui organise des effets d'ensemble sphériques ou à tout le moins courbes, est contrebalancée par la pulsion scopique : le regard colle aux délinéaments, aimant identifier, reconnaître et nommer les choses, les circonscrire, les saisir, les maîtriser. Lorsque le Poète, aux premiers vers du poème, tend la main à un «Voyageur » pour le mener au haut d'une tour afin de lui montrer ce qui s'étend « dans la nuit, à [leurs] pieds, dans l'espace » (109, v. 11), le Voyageur voit d'abord deux choses : « un cercle noir, si large et profond » qu'il « n'en aperçoi[t] ni le bout ni le fond » (v. 13-14) et des « angles noirs et luisants qui, dans l'ombre, / L'un sur l'autre entassés, sans ordre ni sans nombre / Coupent des murs blanchis pareils à des tombeaux 》 (v. 19-21). Le cercle découvert ici se révèle à la dérobée. C'est une forme se manifestant comme une fugace vue d'ensemble dépourvue de contours («ni le bout ni le fond »). Sitôt apparue, elle disparaît : «Des collines, au loin, me semblent sa ceinture », hasarde le Voyageur, parlant du cercle, avant d'ajouter au vers suivant : « Et, pourtant, je ne vois nulle part la Nature, / Mais partout la main de l'homme et l'angle que sa main / Impose à la matière en tout travail humain. » $(109, \mathrm{v} .16-18$.) On constate que la vue d'ensemble se manifeste en creux. Elle s'impose à l'œil par l'absence d'éléments identifiables, par la somme de ce qu'elle soustrait au regard. Elle est ceinturée de masses diffuses qui, peut-être, sont des collines; on ne distingue point son bout ni son fond d'ailleurs comment voir le bout ou le fond d'un cercle, quel qu'il soit? Par définition, il en est dépourvu. En 
revanche, les traits rectilignes qui chaotiquement s'entassent devant la pupille du Voyageur, certes innombrables et désordonnés, s'affirment par le foisonnement; ils invitent à la comparaison; ils opèrent même sur le décor une action, celle de couper les surfaces unies des murs. Leur présence est effective, manifeste et suffisamment nette pour que l'œil sache discerner les formes qu'ils dessinent, tout particulièrement s'il s'agit de droites sécantes, comme le montre la phrase suivante :

Des ombres de palais, de dômes et d'aiguilles,

De tours et de donjons, de clochers, de bastilles,

De châteaux forts, de kiosks et d'aigus minarets;

Des formes de remparts, de jardins, de forêts,

De spirales, d'arceaux, de parcs, de colonnades,

D'obélisques, de ponts, de portes et d'arcades,

Tout fourmille et grandit, se cramponne en montant,

Se courbe, se replie, ou se creuse ou s'étend. (110, v. 27-35.)

Pour reprendre une terminologie que les peintres aiment emprunter à l'univers de la lecture, disons que, dans ce poème, la ligne est lisible (qu'elle soit droite ou non), tandis que les sphères et les volumes à surfaces unies confondent le regardeur. Ils lui posent l'énigme du diffus, du flou, du vague.

La longue énumération des traits, des lignes brisées, des arêtes et des pointes suscite peut-être chez le Voyageur la jouissance de la nomination; la panoplie des angles droits, aigus ou obtus produits par l'industrie humaine n'en demeure pas moins funèbre, semblable aux «tombeaux » de quelque cimetière (109, v. 21). Frappant est le contraste avec la « vivante Roue » $(110, v .45)$, cet « invisible essieu » que « la main de Dieu » « tient et fait mouvoir » (v. 41-42). La vie et le mouvement sacrés sont du côté de l'indéterminé, du vaste, du circulaire et du divin, perceptibles de haut, grâce à un regard embrassant le tout, vision caractérisée par un certain recul. La mort, la fixité s'étendent sur le versant de l'avéré, du détail, de la linéarité et de l'humain, lequel est désigné métonymiquement par la somme nominative des constructions dont il a empli le paysage du moins la part inférieure, terrestre, de celui-ci12.

\section{Manier la règle et le compas}

Le poème de Vigny porte le sous-titre «Élévation13 ». Il s'ouvre sur une insistante invitation à contempler un spectacle grandiose. La première strophe condense en une douzaine de vers les noms « regard », " vue », « œil », et se trouve à itérer par quatre fois la forme impérative du verbe « regarder » (109, v. 1-12). La deuxième strophe se clôt sur la mention d'un trouble de la vision qu'éprouve le Voyageur subjugué par la vaste perspective que l'ascension de la tour lui a permis d'embrasser : « Le vertige m'enivre et sur mes yeux il 
pèse. » $(110$, v. 39.) Quel est ce spectacle hypnotique? C'est celui du moment présent pressenti comme moment historique grâce à la hauteur et au recul que le Poète et le Voyageur ont acquis en gravissant la tour. Et, puisqu'on apprend bientôt que « Le vertige parfois est prophétique » $(111, v$. 74$)$, on dira que ce que les deux hommes cherchent à lire est l'horizon du futur. Ce qu'ils contemplent, c'est autant le grand dessin de la ville que le grand dessein de Dieu : «il n'y a qu'une différence d'une lettre entre "Révélation" et "Élévation” », commente à juste titre André Jarry (1998: 302). Fidèle à la mission qu'il se donne, le poète romantique emmène l'humanité vers les hauteurs célestes à la découverte des intentions divines; il pose en intermédiaire qui sait cueillir aux cieux les présages et les transmettre au peuple grouillant en bas - peuple que lui seul sait « élever » aussi haut.

Cette vocation allégorique du poème pensé comme vecteur d'illumination sociale est renforcée par le fait que le Voyageur et le Poète resteront anonymes. Le premier est un quidam, un étranger à Paris, curieux peut-être d'en apprendre un peu sur la grande ville, enclin à entreprendre un voyage accompagné, une visite guidée 14 qui pourra l'éclairer; son hôte en sait plus long que lui, mais il reste un poète générique, innommé, un indigène familier des curiosités de la capitale, enclin à les présenter au premier venu qui acceptera sa main tendue et qui voudra bien le suivre là où il veut le mener, là où peu d'hommes osent aller. En cela leur « voyage », d'abord d'allure touristique (notamment par l'emprunt à la vogue des panoramas peints $\frac{15}{5}$ ), prend vite un tour initiatique (Jarry, 1998: 306).

Le point de vue enviable qu'ils acquièrent ensemble par l'« Élévation » poétique leur permet d'identifier les signes du passé encodés dans les lignes brisées de l'anguleuse architecture parisienne. Ils décèlent nettement la présence de telle colonnade, de tel pont ou de telle aiguille, marques identifiables du labeur humain accompli à travers les âges. En revanche, le titanesque travail du jour, les machines aux rouages nouveaux, les dynamiques générales qui orientent le monde actuel vers l'avenir restent indéterminés, impossibles à identifier. Bien que le Poète de Vigny soit investi du rôle d'« [i]nterprète des signes » censé bénéficier d'une révélation céleste qu'il transmettra au Voyageur — celle de « la vérité de l'actualité » (Jarry, 1998: 313) —, la vision reste opaque. Paris « se meut » véritablement, d'où peut-être le trouble de la vue, l'étourdissement provoqués : « tout s'ébranle, et tournoie et circule » (Vigny: 110, v. 55). Le « Jaggernaut $\underline{16}$ » (Balzac, 1976 [1834]: 50) qui se fait jour est un rouleau compresseur incarnant la France entière, composée de la somme de ses parties, autant de membres qui lui sont rattachés, puis eux-mêmes imbriqués dans les rouages des pays voisins, « cercles dont la crête / S'enchâsse dans la sienne et tourne sous sa loi » (110, v. 48-49). Ainsi : «Tous marchent leur chemin, et chacun d'eux écoute / le pas régulateur qui leur creuse la route » (110, v. 59-60). Le mouvement est perçu, l'avancée est attestée, mais la direction? mais le but? Et quel nom donner à ces vastes touts? par quel substantif les désigner? Aucun ne semble convenir. Reprenons les vers compris entre nos deux dernières citations : « Le cœur du ressort bat, et presse la bascule; / L'aiguille tremble et court 
à grands pas; le levier / monte et baisse et en sa ligne, et n'ose dévier. » (Vigny, 1950: 110, v. 56-58.) Se dessine certes en filigrane la figure fascinante du moteur à vapeur, dont la présence ne se généralise vraiment qu'à partir des années 1830. Grâce à lui sont conjuguées les forces antagonistes du feu et de l'eau comme jamais auparavant 17 : il les canalise le long de deux droites parallèles, celles du chemin de fer, pour atteindre des vitesses jusque-là inimaginables. Néanmoins, ce que le Voyageur et le Poète entrevoient n'est qu'une vision fugace, indescriptible, tenant de la locomotive qui passe à toute allure devant le badaud ahuri, emportant au loin le secret de ses rouages intimes et de son complexe dispositif de pressurisation. En l'absence d'indications nettes, impossible pour le Poète d'engager son action dans quelque voie $\underline{18}$.

Quoique la tour où le Voyageur et le Poète sont perchés s'élève à l'endroit exact où «Dieu même a posé le centre du compas » lorsqu'll a dessiné Paris sur la carte du monde (Vigny: 110, v. 38), l'œil humain des deux compères ne saurait porter assez loin pour voir l'avenir se dessiner lisiblement. Ils ont peut-être gagné en hauteur et en perspective, ils n'atteignent pas la perspicacité complète dont a pu jouir Dieu. Sans doute ne sont-ils qu'à mi-chemin entre Ciel et Terre. Mais où? Au cœur de Paris, en 1830, on ne peut grimper plus haut que les soixante-dix mètres que font les tours de la cathédrale Notre-Dame - un lieu privilégié, sis en terre

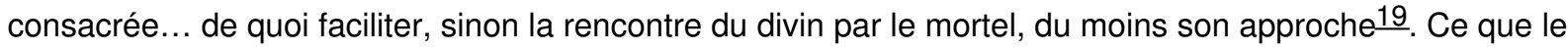
Poète et le Voyageur aperçoivent, néanmoins, n'est pas au-dessus d'eux, mais s'étend sous eux, tout en bas, grouillant, fourmillant. Ils se penchent et découvrent « des travailleurs sans nombre » qui, « Aveugles inquiets, cherchent à travers l'ombre / Je ne sais quels chemins qu'ils ne connaissent pas, / Réglant et mesurant, sans règle et sans compas » (112, v. 120-122). L'humanité a perdu ses repères. Comment s'orienter?

À en croire ces derniers vers, il manque désormais à l'humanité la vue, la lumière, l'assurance, la voie tracée d'avance, jadis procurées par la religion. Pire, lui font défaut les outils du cartographe qui pourraient l'aider à retrouver son chemin : la règle et le compas. La première permet d'évaluer les distances, de chiffrer les intervalles, de tirer des droites régulières; et elle peut servir d'étalon. Mieux encore, le second permet de comparer les longueurs, de les rapporter l'une à l'autre, d'apprécier toutes sortes de coordonnées, de calibrer les diamètres et les rayons; avec lui on lie entre eux les points équidistants d'un centre et on obtient des cercles parfaits. Flaubert près d'un demi-siècle plus tard ironisera, dans son Dictionnaire des idées reçues: « COMPAS : On voit juste quand on l'a dans l'œil. » (1979 [1913]: 500.) Douloureuse condition. II reste que la maîtrise de cet outil, conjuguée à l'application de la règle, offrirait peut-être à nos deux apprentis cartographes la possibilité d'enfin décoder le spectacle d'angles et de rondeurs qu'ils contemplent. Leur lecture des signes inscrits sur la mappemonde étalée sous eux s'en trouverait facilitée. Est-il nécessaire d'ajouter que le mot compas renvoie aussi à la boussole $e^{20}$ ? Voilà un instrument de navigation avec lequel s'orienter dans les vastes étendues privées de repères distinctifs, par sa capacité presque magique d'indiquer en tout temps le nord — celui qu'on perd quand on se dérègle. 
Vigny ne se contente pas d'illustrer la maxime célèbre prononcée par Napoléon fr le 5 juin 1800 devant l'assemblée des curés de Milan : "Une société sans religion est comme un vaisseau sans boussole » (1861 [1800]: 339). II reprend à son compte la part métaphorique de la comparaison proposée par l'Empereur et en fait la clé de lecture du poème. Effectivement, les deux formes antagonistes que Paris donne à voir sous sa plume sont réunies dans le compas : traits brisés autant que cercles unis. La morphologie même de l'outil suggère qu'il est la parfaite incarnation des tensions entre courbe et ligne mises en évidence au sein du poème. Prenez un compas dans votre main et regardez-le; que voyez-vous? Vous pourriez être en train de tenir deux objets différents : ou bien vous voyez deux fines tiges rattachées l'une à l'autre par l'extrémité, c'està-dire deux droites sécantes formant l'image d'une équerre; ou bien vous voyez le sombre cercle de la boussole au cadran hérissé de rayons centrifuges. Dans un cas, l'appareil imite les angles qui envahissent Paris; dans l'autre, son allure cylindrique s'identifie à la forme prise par les effets d'ensemble dans cette ville. Agrandissez maintenant l'échelle des dizaines de fois, des centaines de fois, et posez votre compas géant angle ou cylindre - sur le cœur de Paris comme vous le poseriez sur un plan de la ville étalé sur une table devant vous. Que voyez-vous, sinon la grande Tour où sont perchés le Poète et le Voyageur?

\section{Apostropher Paris}

Le désordre pullulant des hommes s'agitant en tous sens dans la grande ville transfigurée poétiquement par Vigny suggère une idée : Dieu serait-il donc seul à posséder la maîtrise du compas? En tout cas l'univers ordonné, mesuré, quadrillé, orthonormé, qui est celui que les hommes ont bâti en suivant Sa règle et Son dess(e)in des siècles durant, domine l'espace parisien. C'est précisément ce monde-là qui, au tout début de notre analyse, a été identifié comme menacé de sombrer sous les coups aigus de sa propre violence. «Et, comme il est écrit que chacun porte en soi / Le mal qui le tuera [...] » (112, v. 125-126), on peut s'interroger : que reste-t-il de la matière, au bout de tant de heurts? Une fois que la terrible Roue a hachuré tous les segments naguère unis, maintenant jetés au feu de la grande Fournaise appelée à refondre le monde, qu'avons-nous? Vapeurs? Fumées? Le résidu est, en tout cas, quelque chose d'informe :

— Que fais-tu donc, Paris, dans ton ardent foyer?

Que jetteras-tu donc dans ton moule d'acier?

Ton ouvrage est sans forme, et se pétrit encore

Sous la main ouvrière et le marteau sonore;

II s'étend, se resserre, et s'engloutit souvent

Dans le jeu des ressorts et du travail savant [...]. (113, v. 185-190.)

Nous touchons ici presque à la fin du poème. Le Poète en quête de précisions sur le futur peut bien apostropher Paris, il n'aura, pour toute réponse, qu'un « sourd mugissement » montant à lui « du centre de la 
France / [...] Apporté par le vent des nuages glacés » (114, v. 196-198). N’empêche, ce " grand silence » (114, v. 195), qui remet en cause les facultés divinatoires dont le Poète s'estime détenteur, distrait notre attention d'un fait : les clés de l'énigme se trouvent inscrites à même les interrogations adressées à la vaste cité personnifiée. Relisons l'apostrophe faite à Paris. On y trouve la « main ouvrière », le « marteau », le « travail savant », la détente du « jeu des ressorts »... L'avenir reste à venir, mais clairement il ne sera pas façonné par la main de Dieu. Il appartient aux hommes. À leur savoir et à leur industrie.

On peut bien conclure avec André Jarry que « [l]a "force divine" est appelée à émigrer — en l'homme 21 » (1998: 311), il faut quand même reconnaître qu'il y a là une dégradation. Elle est assortie d'une inquiétude tenace; celle d'échouer, faute du secours divin. Qui sait si, malgré l'aspect christique de la mission qu'assigne Vigny aux poètes dans les derniers vers du texte (Bénichou: 146), la règle et le compas maniés librement par les hommes nouvellement affranchis de Dieu n'iront pas, « labourant le Globe comme un champ », semer « la douleur du levant au couchant; / Rasant l'œuvre de l'homme et des temps [...] » (113, v. 173-175)?

L'alternative à cette autodestruction paraît pis encore. Elle est portée par la peur sainte qu'éprouve le Voyageur, sorte de version 1830 du valet mis au service du dialogue philosophique avec son maître $\underline{22}$. Lorsque, s'étant sans doute " élevés » trop loin ou trop profondément, lui et son guide se sentent perdre la tête (Vigny: 114, v. 199-206), il recule avec effroi : « la hauteur où nous sommes / De corps et d'âme est trop pour la force des hommes! » (114, v. 202-203.) S'instille en lui une inquiétude tenace. Qu'adviendrait-il si Dieu, non pas disparu mais seulement resté passif un temps, s'était laissé irriter par la prétention de l'humanité à s'élever aussi haut que Lui? Se préparerait-II, maintenant, à déchaîner Son juste courroux? Grossissant soudain au beau milieu du ciel s'esquisse alors une forme douteuse : "Quelque chose de noir, de lourd, de vaste, là » (114, v. 210). S'agit-il de « ce rocher ténébreux / Qu'annoncèrent jadis les prophètes hébreux » (114, v. 213-214), ce terrible obus aux mille arêtes censé rayer de la carte "la ville enivrée / D'ellemême » (114, v. 219-220), afin de nettoyer la société autolâtre? Ce serait l'exécution d'un châtiment divin longtemps attendu : l'Apocalypse. Mais, en croyant déceler dans le ciel noir de la pleine nuit, derrière un nuage noir, la silhouette de la « meule énorme » $(114, v$. 215$)$ du mythe biblique, ce Voyageur ayant jusque-là paru très neutre se révèle plutôt opiniâtre et enclin à voir ce qu'il veut bien voir dans ces ténèbres quasi complètes $\underline{23}$. Effrayé par le spectre de la fin des temps, il s'affirme en chrétien vindicatif, soucieux de ce que Pierre Citron appelle « les excès des Trois-Jours » (275) et somme toute réfractaire à la modernité24.

Par chance, ce terrible «Rocher du ciel » (v. 222) pourrait s'avérer n'être qu'un simple nuage de vapeur, produit - pourquoi pas? - par l'intense activité de la fournaise moderne, ce « feu de la pensée » (Drissa: 118) destiné éventuellement à faire avancer la machine infernale du temps. Ne nous 
rassurons pas trop vite. Même un inoffensif nuage reste un mauvais augure : le futur à déceler approche, mais il s'affiche voilé, diffus, sans contours nets, indécis, potentiellement meilleur que le passé qu'on connaît si bien; possiblement plus sombre aussi, ou plus étouffant. Bref, rien n'est sûr. Et le pouvoir divinatoire de la poésie semble avorter 25 . Sans en apprendre davantage, les deux hommes terrifiés n'osent plus deviner quoi que ce soit. Ils se résignent à « quitt[er] cette imposante cime » penauds et inquiets (115, v. 235) et n'emportent de leur aventure qu'un plaisir ineffable : celui, esthétique, d'avoir tant entrevu de choses.

Nulle philosophie de l'histoire ne triomphe dans ce poème du devenir, ce qui en soi est un parti pris éloquent, philosophiquement significatifㅡㅡ. La critique littéraire des dernières décennies a amplement établi que la modernité qu'inaugure Vigny en poésie, et peut-être aussi en philosophie, est celle d'offrir en partage sa part d'illisibilité au texte poétique, lequel vient à s'imposer en «mystère inviolable » (Bertrand: 83) porteur de vertige et d'interrogations irrésolues. Le trouble de la vision et l'ébranlement de la pensée qui affectent le Poète et le Voyageur en haut de la tour, malaises générés l'un par l'autre et se relayant, s'aggravant l'un l'autre, n'ont d'égal que ceux éprouvés par le lecteur du poème lui-même, qui devra lire, relire, imaginer, repérer, s'arrêter, supposer, interpréter, comparer, réexaminer, déceler, conjecturer, s'il veut goûter le texte et orienter sa compréhension, invinciblement partielle $\frac{27}{}$, du « Paris » qui y est proposé. Dans ces conditions, regarder, penser, soupeser, saisir des yeux sur papier cette grande ville emblème d'un nouvel ordre humain, couchée en caractères noirs cordés sous nous, comporte un risque que nous ne voulons nous empêcher de prendre et qu'heureusement nous pouvons reprendre à l'infini : celui de lire et de se tromper, de ne pas bien voir, de ne pas suffisamment comprendre, de mal démêler le chaos des lignes, de s'y empêtrer et d'avoir à recommencer pour affiner ses vues. C'est le même risque qu'ont pris les Parisiens trimant dur au pied de la Tour dans ce poème, comme le remarque Pierre Citron : «Paris est le symbole de la seule espérance possible pour I'humanité : celle du progrès de la pensée. Paris est un danger qui doit être couru. » (277.)

\section{Avancer malgré tout}

Si la poésie de la grande ville moderne ne saurait nous dire précisément où va Paris, Vigny inscrit tout de même assez d'indices dans son poème pour que, voyant le passé et constatant le mouvement actuel, nous puissions déduire les principales orientations qui président à l'avancée de ce grand cercle noir qui est partie « Roue », partie « Fournaise » (110, v. 40). Évidemment, reculer n'est pas une option. La roue tourne sous l'effet de la fournaise et l'action de la roue alimente la fournaise; le tout s'emballe dans une infernale accélération que le lecteur est invité à suivre.

À travers le rétrécissement de Dieu à hauteur d'homme s'établit, dans le «Paris » de Vigny, une association entre, d'une part, l'univers particularisé des arêtes régulées par la mesure du compas divin, et, d'autre part, celui de l'action humaine qui, à travers les siècles, a érigé tant de monuments en Son honneur. L'architecture 
parisienne, telle une forêt, hisse ses cimes vers le ciel (110, v. 30); elles ont monté progressivement, année après année plus hautes, inondant petit à petit le ciel de leurs innombrables lignes brisées. L'air vital s'y raréfie et Dieu avec lui. Mais la grande absente du tableau est une autre divinité, pourtant particulièrement chère à l'esprit romantique depuis Rousseau : la Nature. Elle paraît avoir été matée par la main de l'homme, étouffée par la profusion des angles que celui-ci, dans sa volonté de régir le monde selon les préceptes divins, a partout appliqués (109, v. 16-18). On l'a réduite à servir de support aux constructions humaines. Surplombant « cet abîme où l'air pénètre à peine ", le Voyageur compare les lanternes et réverbères allumés dans la nuit à « des diamants incrustés dans l'ébène » (109, v. 23-24). L'image est éloquente. Des pierres puisées au plus intime de la croûte terrestre, pour être ensuite taillées avec force, aiguisées avec précision, polies à l'envi, ont été serties minutieusement entre les nœuds, les veines, les fibres mêmes d'un morceau de bois jadis poussé à même cette terre, sous forme d'arbre bercé par les vents, chauffé par le Soleil, qui éventuellement a été abattu, puis découpé, mesuré, poncé.

Comment mieux dire la sublime violence transformatrice dont l'homme s'est fait l'agent en appliquant aux tortueux et irréguliers substrats naturels le tranchant, la pureté et l'éclat des rectitudes idéales qui sont le fond de la règle divine? Belles à regarder, ces constructions anguleuses chamarrées d'arêtes peuvent avec le recul devenir effrayantes à contempler. On comprend que «Les rayons effrayés disent au cercle : Arrête. » (110, v. 47.) Vœu pieux, prière qui ne sera jamais entendue.

\section{Mater les batraciens}

II n'y a réellement qu'un seul élément naturel du paysage parisien qui, au fil des siècles, semble avoir échappé à l'emprise de la règle et du compas. Ou plutôt dirons-nous qu'il s'en est accommodé, qu'il s'est plié à eux mais a su rester retors. On le rencontre dès les premiers vers du poème. C'est une ligne : non pas droite, mais courbe, sinueuse. Elle est nettement dessinée tout juste sous la Tour; pourtant, à gauche comme à droite, elle fuit vers l'infini des lointains. II s'agit donc d'un hybride alliant de façon intrinsèque les atouts de la surface et du trait, d'où sans doute son statut d'exception. À cette ligne sulfureuse le regard du Voyageur et du Poète « s'attache avec feu, comme l'œil du serpent / Qui pompe du regard ce qu'il suit en rampant » (109, v. 5-6). Elle est mouvante, aussi, puisqu'elle se colle au pied du donjon, tourne sur lui, s'y enroule (109, v. 7). En même temps, elle paraît attachée à ce « moyeu divin » $(111$, v. 68), telle une bête menaçante enchaînée à son poteau, retenue, contenue par lui. À l'instar de la plupart des objets courbes dans ce poème, cette ligne reste innommée. Néanmoins on l'identifie sans peine : «- Un fleuve y dort sans bruit, replié dans son cours, / Comme, dans un buisson, la couleuvre aux cent tours. » (109, v. 25-26.) Les serpentements de la Seine l'associent d'emblée à l'imaginaire de la tentation diabolique, d'où les « cent tours » qu'elle peut nous jouer.

Pour être prévisible, ce sempiternel rapprochement avec le serpent tentateur de la Genèse se trouve en 
quelque sorte relayé ou à tout le moins légitimé, au $\mathrm{XIX}^{\mathrm{e}}$ siècle, par la situation prévalant aux abords du fleuve parisien, caractérisés par le vice des bas-fonds de la société (Kalifa: 10) : le dur labeur des porteurs d'eau, des bateliers, des débardeurs, des tireurs de sable, y côtoie le danger imprévisible des crues $\underline{28}$ ainsi que l'infection des milieux humides $\underline{29}$, vaseux, peu fréquentables, infestés d'insectes, de lézards, de batraciens, de vermine et de gens louches, tous symboles séculaires de comportements lestes et d'immoralité. $Y$ foisonnent, vers 1830, tant le péché $\underline{30}$ que les miasmes 31 ou la saleté 32 . Ces maux de l'âme et du corps, la chrétienté s'est targuée de les éliminer à Paris dès le haut Moyen-Âge en érigeant des églises, en balisant le territoire, en étendant son emprise jusque sur les terres inondables du bassin fluvial. Comme l'explique l'historien André Guillerme, l'Église s'est imposée en région parisienne grâce à une lutte contre les menaces propres aux berges de la Seine : inondations, certes, mais aussi criminalité, pauvreté, maladies.

Exemplaire à cet égard est le cas de saint Marcel, évêque des débuts de l'ère chrétienne, dont la légende raconte qu'il a terrassé un dragon sévissant non loin de la Cité, à l'embouchure de la Bièvre. Jacques Le Goff (1991) a étudié la fortune de ce motif hagiographique, qui, selon lui, remonte aux origines de la tradition chrétienne et trouve sa logique dans l'élan de christianisation emportant l'Occident médiéval. Le plus souvent, l'hagiographie met en scène le combat d'un « évêque dompteur » contre un « monstre apprivoisé », sur fond de service civique (Le Goff: 256). En d'autres termes, l'affrontement constitue moins un duel à mort qu'un domptage de fléau local; c'est quelque force naturelle à brider, un secours social à mettre en œuvre, un écueil commun à atténuer sinon éradiquer (Le Goff: 243). II faut insister, plaide Jacques Le Goff (241), sur I'homologie et même sur l'équivalence symbolique qui s'instaure dans la chrétienté médiévale entre dragon et serpent au sein de ces récits où le Mal finit toujours vaincu par le chef de la congrégation : "Ce dragon, c'est le serpent de la Genèse, c'est le vieil ennemi de l'homme, c'est le diable, c'est Satan » (246). Mais ce qui nous intéressera par-dessus tout, dans cet héroïsme chrétien, c'est l'outil avec lequel l'évêque parvient habituellement à écraser son adversaire; car, plus souvent qu'autrement, le geste décisif, celui qui mate la bête légendaire, consiste à enfoncer la crosse épiscopale dans la gueule du dragon (Le Goff: 265). D'ailleurs l'ornementation de ces bâtons sacrés s'en fait l'écho: « Rares sont les crosses épiscopales qui ne retiennent captives en leur tête recourbée le dragon vaincu » (261), observe Jacques Le Goff. Elles-mêmes hybrides, ces crosses droites au bout recourbé, symboles de la puissance de l'évêque, servent d'arme capable de contenir le vice, de cerner le mal, d'amadouer l'adversité — comme de lier d'un trait l'évêché au Saint-Siège et la terre ferme aux nuées célestes.

Marcel protecteur de Lutèce fut longtemps premier parmi les saints patrons de Pari33 3 . Or, trois des cinq miracles lui étant attribués ont pour objet principal l'eau : sa conduite, sa transformation, sa multiplication $\underline{34}$. Son principal fait d'armes — l'affrontement avec le dragon — a pour cadre géographique les marécages de la basse vallée de la Bièvre, au confluent du fleuve. Le grand saurien incarne un condensé des problèmes 
associés aux marécages de la périphérie des cités : à terme, « [l]a zone humide est asséchée et mise au pas chrétien » (Guillerme: 39). En sorte que, quand Vigny parle du compas que Dieu aurait appliqué sur le globe pour dessiner Paris, une perspective sensible au folklore et à la culture ecclésiastique propres aux bords de Seine et tout particulièrement à Notre-Dame $\frac{35}{}$, permet d'y voir un geste d'une certaine violence : avec la pleine autorité qui lui revenait jadis, Dieu aurait planté la pointe effilée de son compas dans la Seine, épinglant ainsi le dragon d'eau dans son cours définitif.

Nous voilà devant une nouvelle dégradation : de la sainte crosse ornementée, cisaillée, consacrée, on aboutit au compas laïc, mathématique, droit comme un I, épuré, sans fioriture aucune. Ce Voyageur a beau être fervent chrétien, la vue de Paris ne lui a pas inspiré l'image, fidèle à l'hagiographie traditionnelle, d'un dieu servi par ses évêques " héros sauroctones, libérateurs et civilisateurs 》 (Le Goff: 256); mais celle de Dieu privé du recours de ses serviteurs, maniant son compas seul contre le dragon. Ce qu'on perd en panache et en sacralité, on le gagne peut-être en précision et en syncrétisme.

Dieu naguère voyait clair dans le jeu liquéfiant des forces du mal qui toujours menacent d'engloutir les hommes; II a employé sa règle et son compas à mater les dragons en soumettant ces derniers aux meurtrissures de la ligne droite et de la mesure. Cependant, à la fin du poème, Dieu réformé n'a plus de main pour frapper, d'où le retour en force des batraciens, emblèmes chrétiens du péché : « Salamandres partout!... Enfer! Éden du monde! », s'écrie le Poète (113, v. 156). II ne faut pas croire que ce pullulement apparemment nouveau vient s'ajouter ou se mêler à celui, préalablement cité, des hommes au travail; ils sont ici une et même chose. Toutefois, ce vers semble évoquer la possibilité que l'omniprésence du péché humain se transmue en paradis terrestre. L'antithèse enfer-éden que porte le second hémistiche se fait interface paradoxale entre deux mondes aux antipodes. Elle surprend; à y regarder de près, elle ne paraît autorisée que par l'ambivalence symbolique de la salamandre. Mi-grenouille par sa peau, mi-serpent par sa longue queue et son corps fuselé, ce furtif quadrupède amphibie aux allures de petit mammifère fait figure d'oxymore. II sait courir comme un rongeur, nager comme un poisson, glisser comme un lézard... Le folklore lui prête une résistance aux incendies et même le pouvoir de les éteindre, en plus de nombre d'autres vertus ésotériques (Tervarent: 388-389). Dragon de feu, dragon d'eau, il orne les armes de la ville du Havre, sise à l'embouchure de la Seine; il orne de même le blason de François $1^{\mathrm{er}}$, grand roi de France dit le « Prince de la Renaissance » : recourbée sur elle-même, sa silhouette se détache, sur fond bleu aquatique, au milieu des flammes ardentes d'un brasier.

Créature familière des bestiaires médiévaux et de l'héraldique française, la salamandre est un dragon diminutif et surtout naturel, un être moins formidable par sa puissance qu'ambigu par sa polymorphie et sa fugacité; elle allie, ainsi que la machine à vapeur, les dynamiques imprévisibles et antagoniques sur lesquelles la modernité 
prétend s'édifier. Plus que tout, loin d'incarner, comme son titanesque grand frère le dragon, une menace à contenir, elle symbolise plutôt un bienfait, quelque pouvoir spécial, ou se veut porteuse d'auspices favorables. Sous cet angle, les forces vives vautrées dans les miasmes du péché débridé qu'elle représente dans le poème prennent la valeur d'un trésor national à chérir, car issu de lignée royale, hérité de l'esprit humaniste de la Renaissance, qui le premier porta atteinte à l'hégémonie de l'Église, et relayé de siècle en siècle par la parole populaire, par l'imaginaire vif, hardi et leste du peuple, clé de voûte des utopies progressistes des années 1830.

Les hommes nés égaux peuvent se réjouir de ce que «la force divine » ait été intériorisée et se trouve dorénavant détenue collectivement « en ceux dont l'esprit sent, prévoit et devine », selon la formule de Vigny (114, v. 227-228). Sans doute est-ce à eux, maintenant, qu'il faut s'en remettre pour se guider à travers la vallée des tentations? Cependant, le Poète et le Voyageur ne semblent pas donner cher de l'avenir. Si le grand serpent reste sagement cloué à leurs pieds, l'enfer paraît quant à lui être remonté sur terre pour inonder l'espace. À vrai dire, ce n'est pas le royaume de Satan tout entier qui reflue, mais seulement le chaos, lequel n'en est qu'une modalité. Ce bouillonnement à la surface a laissé, au fond des fosses abyssales, les boues de l'immoralité; en se délestant de la dimension religieuse qu'il a toujours charriée, l'enfer est devenu simple tumulte. Risqué sûrement, fécond peut-être, il n’est plus qu'activité désordonnée, débridée, potentiellement céleste.

On serait tenté d'en inférer que, dans ces conditions, aucune certitude ne subsiste. N'assimilons pas la pensée de Vigny à un nihilisme simplet et anachronique qui jamais ne fut le sien. Car, mis devant le nuage sombre qui se profile à l'horizon, symbole même de cette existence chaotique, privée de la providence divine, le Poète ose, en dernière instance, avancer une image attendue. C'est celle de la ligne de vie, sur laquelle il identifie deux points incontournables :

— Je ne sais d'assurés, dans le chaos du sort,

Que deux points seulement, LA SOUFFRANCE ET LA MORT.

Tous les hommes y vont avec toutes les villes. (115, v. 237-239.)

Ainsi, Vigny au bout du compte nous invite à tracer la ligne, non pas droite, mais sinueuse, qui relie l'un à l'autre ces deux points énoncés en petites capitales, les seules du poème. Ce parcours capital, et communément partagé par tous, se dessine désormais sur la carte du monde à main levée, sans le recours des balises, sans le secours de la mesure. Force est de l'imaginer ondoyant, impétueux, tordant son flanc d'un côté, se recourbant vers l'autre, contournant les obstacles, se précipitant à travers les cascades, dévalant des 
rapides, s'engluant dans les sables, ralentissant aussi en de paresseux méandres, avant de se jeter dans l'Océan, terme obligatoire d'un trajet ponctué de heurts, d'allers-retours, de culs-de-sac et d'avancées.

L'usage des petites capitales est typographiquement significatif. II signale, dans l'immense étendue fluide des petits caractères arrondis (et indifférenciés) qui peuplent les pages du poème, deux points d'ancrage distinctifs, plus carrément posés, plus massifs, plus droits, plus hauts. Plus anguleux aussi, et tranchants : c'est bien de la souffrance et de la mort qu'il s'agit. Ils marquent au stylet, tel la pointe d'un compas appliqué avec trop de véhémence à travers le papier, les deux abîmes où le poète romantique pourra verser ses principales ressources mentales. Inversement, mais c'est la même chose, ces puits sans fond sont le fonds noir où il pourra tremper sa plume et du haut desquels il pourra étendre l'encre de son sentiment sur la surface des choses étalée sous lui. Car la souffrance et la mort, dont il détient une conscience aiguë, sont pour lui deux tours d'ivoire qui l'élèvent au-dessus de la mêlée. C'est à partir de là qu'il est habilité à servir d'intermédiaire aux autres et c'est pour cela qu'il s'autorise à coucher, pour le bénéfice d'autrui, l'une après l'autre des lignes parallèles de caractères, petits bâtons d'ébène mesurant tous le même nombre de pieds, rendus scintillants par les cent tours de magie que la pensée bien coulée, subreptice et brillante, sait jouer au lecteur quand les caractères lus s'effacent sous les yeux pour former la vision poétique.

Qu'est-ce en effet qu'écrire de la poésie, vers 1830, sinon donner forme à l'informe par la lignę6 ? C'est pourquoi on ne peut rester aveugle à la teneur métapoétique du compas, image phare de notre analyse : ne sert-il pas à diviser par segments égaux les droites, à calculer avec précision le nombre de mètres ou de pieds qui séparent deux localisations sur la carte? L'œil qui parcourt les vers correctement alignés sur la page serpente de gauche à droite, recommençant de ligne en ligne, pompant « du regard ce qu'il suit en rampant » (Vigny: 109, v. 6), happé par ce qu'il ne voit pas mais qu'il visualise en lisant, incapable de décrocher de sa vision.

1. II ne s'agira pas pour nous d'examiner à nouveaux frais le rôle de précurseur joué par Vigny à l'égard de la sensibilité poétique inédite qu'impose la grande ville moderne, telle que mise en évidence chez l'auteur des Fleurs du Mal par Walter Benjamin (1989). On pourra se reporter à ce propos aux éclairantes remarques de Jean-Pierre Bertrand et Pascal Durand (84-86).

2. Outre-Manche, l'industrialisation fut plus rapide et plus importante (Laforgue: 63-64).

3. La loi électorale votée le 19 avril 1831 est l'aboutissement de débats à la Chambre des députés en cours depuis février de la même année (Tudesq), et ce, dans la foulée des soulèvements républicains à Paris des 14-15 février, qui ont vu l'église SaintGermain-l'Auxerrois et l'archevêché de Paris mis à sac, suite au service funèbre donné par les Légitimistes en rappel de l'assassinat du duc de Berry (Baldensperger: 249). Si la plaquette de Vigny parut le 16 avril 1831, le poème fut vraisemblablement écrit entre novembre 1830 et la mi-janvier 1831 : Vigny l'a daté ou du 6 janvier (Baldensperger: 249) ou du 16 janvier (Jarry, 1998 : 305, n. 258), dates qui, l'une comme l'autre, sont forcément approximatives (Jarry, 1973: 289). Il est établi en tout cas (Jarry, 1998: 305) que Vigny lut le poème à Montalembert le 23 mars 1831, quelques semaines seulement avant de le faire paraître. L'actualité politique, même la plus immédiate, a donc pu teinter, ne serait-ce que superficiellement, la version publiée — ou encore en motiver une publication « à chaud », mûrie moins longtemps que de coutume pour l'auteur (Jarry, 1998: 305, n. 260). 
4. Notre-Dame de Paris de Victor Hugo paraît le 17 mars 1831, quelques semaines avant le poème de Vigny. Deux ans plus tôt, Prosper Mérimée avait publié sa Chronique du règne de Charles IX (1829).

5. Les premières pages de Stello paraissent dans la Revue des Deux Mondes dès le 15 octobre 1831.

6. En 1830, le mythe de Paris existe à peine. Si les premiers linéaments de ses principales composantes sont dessinés, leur synthèse n'a pas été faite [...]. En 1840, tout est fait; la rhétorique de Paris a pris corps [...]. » (Citron: 319.)

7. L'originalité d'un tel sujet est complète selon Pierre Citron : " pour la première fois en vers sont unis ici le thème de la nuit dans la ville et celui du panorama parisien » (269).

8. Dans la version de 1837 du Chef d'œuvre inconnu, remaniée et bonifiée, le peintre Frenhofer affirme qu'« il n'y a pas de lignes dans la nature où tout est plein » et que " [l]a nature comporte une suite de rondeurs qui s'enveloppent les unes dans les autres » (Balzac, 1979 [1837]: 424). Ce conte avait paru pour la première fois en deux livraisons dans L'Artiste, les 21 juillet et 7 août 1831 , toutefois les remarques de cette teneur sont absentes de la préoriginale, très dépouillée; elles n'apparurent qu'avec les amendements ménagés pour l'édition des Études philosophiques de 1837 chez Delloye et Lecou, laquelle voit le texte doubler en volume par l'apport d'un discours esthétique et technique emprunté pour une large part, selon Pierre-Georges Castex, à Denis Diderot, que Balzac vers la fin de 1836 a relu (1979 [1837]: 1407). Pierre-Georges Castex relève au sein du vocabulaire pictural des Essais sur la peinture (1765) le mot de " coloriste " (Balzac, 1979 [1837]: 1416), que Balzac prête à Frenhofer; il cite en outre la façon dont Diderot, dans ses Pensées détachées sur la peinture (1776), théorise l'opposition ligne-couleur (Balzac, 1979 [1837]: 1416, 1421-1422).

9. À Delacroix Baudelaire pardonne volontiers « des fautes de détail et des taches microscopiques », lorsque « [l]'ensemble est si beau » (1999 [1846]: 171).

10. Baudelaire, qui a bien choisi son camp, argue notamment que « la touche mangera toujours la ligne » et plaide que «le choix de sujets à mouvement [exige] l'usage des lignes flottantes et noyées » (1999 [1846]: 152). Ce faisant, il dramatise volontiers l'affrontement entre les deux clans : "Les coloristes dessinent comme la nature; leurs figures sont naturellement délimitées par la lutte harmonieuse des masses colorées. / Les purs dessinateurs sont des philosophes et des abstracteurs de quintessence. / Les coloristes sont des poètes épiques. 》 (1999 [1846]: 152.)

11. Par cette formule, le critique Maxime Gaucher cherche à ridiculiser la vision par trop manichéenne que les romantiques voulurent imposer à l'encontre de leurs prédécesseurs : «La couleur représente, paraît-il, l'aspiration vers l'infini, la spiritualité. J'avais cru, au contraire, l'art classique profondément spiritualiste et je concevais même jusqu'à un certain point qu'on lui reprochât de l'être trop exclusivement. » (Gaucher: 971.)

12. Toute terre-à-terre qu'elle puisse paraître dans ce passage descriptif du poème, la ville dépeinte par Vigny intègre certains détails fantaisistes qui, nous éloignant du Paris réel, ouvrent à l'imagination et à la généralisation, comme l'a fait remarquer Pierre Citron : «Vigny semble [...] avoir voulu y mêler plusieurs villes : une cité féodale avec ses donjons, ses châteaux forts et ses remparts [...]; une ville orientale avec ses kiosks, ses minarets, ses obélisques, ses dômes; une ville classique avec ses palais, ses jardins, ses parcs, ses colonnades; le tout aboutit à une ville fantastique, qui, bien que toute de pierre, toute en angles, privée de tout élément naturel, rejoint pourtant par certains aspects le foisonnement de la nature : on y distingue des forêts; ville en relief dont la géométrie s'organise avec insistance dans trois dimensions, et non sur un plan comme il était traditionnel; et surtout ville mouvante, grouillante [...] : ville où les maisons et les édifices semblent avoir une vie propre, qui se superpose à la vie des hommes tout en la symbolisant. " (269-270.) On notera que le transport de l'Obélisque de Louxor à la place de la Concorde, prévu depuis le début 1830 - avant la Révolution de Juillet — n'a été complété qu'en 1836. Vigny cède ainsi à l'orientalisme romantique tout en décrivant une cité future, non encore advenue, mais en passe de se réaliser.

13. En 1837, Vigny rebaptise Poèmes antiques et modernes son recueil de Poèmes qu'il réédite en l'augmentant périodiquement de nouvelles pièces depuis 1822. La mouture de 1837 intègre deux longues pièces sous-titrées «Élévation »: “Les amants de Montmorency » et «Paris », qui, rédigées après la plupart des autres (respectivement en 1830 et 1830-31) et publiées d'abord séparément (en 1832 et 1831), viennent clore désormais le livre sur une note résolument moderne. À l'origine, l'auteur les destinait à paraître dans un recueil intitulé Élévations, dont le projet a été abandonné. Une lettre adressée par Vigny à Camille Maunoir le 26 décembre 1838 nous renseigne sur le sens qu'il donnait à ce mot : « J'ai nommé ces poèmes Élévations parce que tous doivent partir de la peinture d'une image toute terrestre pour s'élever à des vues d'une nature plus divine et laisser (autant que je le puis faire) l'âme qui me suivra dans les régions supérieures, la prendre sur terre et la déposer aux pieds de Dieu. 》 (Vigny cité par Jarry, 1973: 288.)

14. Le poème entier se présente comme un entretien à deux autour d'un même objet de curiosité : un panorama de Paris vu de haut. II s'agit cependant d'un dialogue « dissymétrique ", comme l'a remarqué André Jarry (1998: 306) : jamais nommé ni même désigné explicitement comme «Poète », le « héros » (Citron: 268, n. 6) du poème agit comme « narrateur » (Jarry, 1998: 306); il tutoie son compagnon, qui, lui, le vouvoie; il dirige le couple en haut de la tour comme il dirige les réflexions qui sont échangées; il détient le privilège de prononcer le premier et le dernier mot du poème; il obtient un temps de parole quatre fois plus important que celui du Voyageur.

15. Sur le dispositif scénique du panorama, estrade circulaire où le visiteur monte contempler une fresque peinte en trompe-l'œil (l'entourant sur 360 degrés), voir la note que proposent Jean-Pierre Bertrand et Pascal Durand (84, n. 110). Balzac emploie l'image dans Le Père Goriot pour parler du Paris moderne : « Le char de la civilisation, semblable à celui de l'idole 
16. de Jaggernaut, à peine retardé par un cœur moins facile à broyer que les autres et qui enraye sa roue, l'a brisé bientôt et continue sa marche glorieuse. " (50.) Aujourd'hui on dirait plutôt Juggernaut, d'après le mot anglais lui-même dérivé du sanskrit Jagannath, nom du temple de Purî en Inde, lié aux processions de grands chars surmontés d'idoles et réputés (selon la légende coloniale) écraser tout ce qui se trouve sur leur passage, y compris les personnes, les bêtes. On soupçonnait même les fidèles de se jeter sous les roues du char promenant la statue du dieu Vichnou pour accéder à une caste supérieure dans leur prochaine vie.

17. C'est à ce paradoxe physico-chimique que l'on peut, à la suite du sociologue Alain Gras (2007: 95), attribuer l'impact capital de la machine à vapeur sur l'imaginaire social de l'époque : « Le capitalisme thermo-industriel va utiliser l'eau au service de la puissance du feu, créant une rupture dans l'équilibre des éléments naturels. En principe, l'eau et le feu doivent être séparés [...]. L'eau éteint le feu [...] : pour la première fois l'eau est enfermée dans un espace clos, réduite à l'état de servante du feu. 》 (92-95.)

18. Sur la question précise de l'engagement poétique, voir la démonstration biographico-psychanalytique d'André Jarry (1998: 313314).

19. Il convient de mentionner que plusieurs critiques (Flottes: 79; Baldensperger: 249; Drissa: 115) ont assimilé la tour du poème à une figuration imaginaire des cent trente mètres de la butte Montmartre, où Vigny se rendait régulièrement. Toutefois, le choix d'un tel axe se trouve à excentrer de beaucoup le point de vue des deux protagonistes du poème, trop pour qu'il corresponde au centre de la ville : Montmartre n'est pas Paris en 1830; c'est une commune autonome qui ne sera rattachée à la Capitale qu'en 1860. Quoi qu'il en soit, la butte constitue elle aussi un important lieu de culte chrétien pour les Parisiens, et ce, depuis le Moyen-Âge (la construction de la basilique du Sacré-Cœur, entamée en 1875, n'a qu'accentué une réalité déjà bien en place). Quant à la tour Saint-Jacques, clocher gothique flamboyant du $X V l^{e}$ siècle érigé tout près de Notre-Dame (mais sur la rive droite), il est significatif qu'elle ait servi de tour à plomb pour une fonderie entre 1824 et 1836 (Lambeau, 1906: 268). L'image de la fournaise, si importante dans le poème de Vigny, favorise en un sens l'association de la tour imaginaire gravie par le Poète et le Voyageur à celle-là. Or, parce qu'elle culmine à seulement cinquante-quatre mètres, soit une quinzaine de moins que les tours de Notre-Dame, et parce que son capital symbolique, en particulier à la période romantique, est nettement moindre que celui de ces dernières, il paraît peu probable que la tour Saint-Jacques incarne le sommet parisien dont il est question dans le poème de Vigny (Citron: 267). On doit plus vraisemblablement situer l'endroit où sont montés le Voyageur et le poète à l'emplacement de Notre-Dame, sur l'île de la Cité, enlacée par les deux bras de la Seine, véritable cœur de Paris aux yeux du XIXe siècle. II faut, du reste, se rallier à l'opinion de Pierre Citron, qui plaide contre « toute localisation précise » d'une tour plus imaginaire que toutes celles que Vigny aurait pu sans difficulté désigner nommément : «Vigny écrit [...] Tour avec une majuscule au premier vers du poème, donnant ainsi à l'édifice une valeur générale et intemporelle. À moins qu'il ne veuille suggérer une imaginaire Tour de Babel? »(Citron: 268.)

20. La synonymie est attestée depuis la Renaissance.

21. Jarry cite Vigny (1950 [1831]: 114, v. 227).

22. La tradition est faste, du Glaucon de la République de Platon au Jacques le fataliste de Diderot.

23. La volonté paradoxale et sublime de tâcher d'appréhender ou même de décrire ce que l'on ne perçoit pas est l'une des caractéristiques récurrentes de la poésie de Vigny, souligne Bérangère Chaumont, laquelle a relevé « la prédilection du poète pour une scénographie nocturne »(66).

24. Pierre Citron (276) signale que cette attitude réactionnaire éloigne le Voyageur de celle, plus posée, du Poète, tout en le rapprochant de Vigny lui-même, de la part plus crédule, plus anxieuse et plus véhémente aussi que ce dernier porte en lui.

25. "Le poème, en quelque sorte, s'annule lui-même », observe à ce propos André Jarry (1998: 311). Jean-Pierre Bertrand et Pascal Durand nous convient d'ailleurs à saluer Vigny comme l'un des premiers à avoir fait du poème « le lieu d'une interrogation explicite de la poésie » (59).

26. Notons que cette posture adoptée par Vigny constitue pour lui une phase de transition, liée au choc éprouvé face aux événements de 1830. Si elle ne durera pas (Jarry, 1998: 314), elle ouvre la poésie, d'après Jean-Pierre Bertrand et Pascal Durand, à un nouveau régime esthétique : «La ville objet d'un texte, objet textuel et figure générale de l'engendrement du texte : sans en prendre probablement toute la mesure, Vigny fait là un choix qui apparaît, rétrospectivement, comme un acte d'instauration de la modernité même. Cet acte restera, chez lui, sans suite et à plus d'un titre ses poèmes philosophiques se tiendront en retrait de l'ambition qui un instant aura été la sienne de forger un langage en résonance avec l'époque. 》 (86-87.)

27. «Modernes seront les textes ayant au surplus cette propriété de reculer indéfiniment l'horizon de la chose représentée - le monde étant donné comme un texte - et de conférer à leur propos une dimension spéculaire ", commentent Jean-Pierre Bertrand et Pascal Durand (83).

28. D'importantes inondations surviennent sporadiquement à Paris sans doute depuis toujours, emportant chaque fois des ponts, des quais, des maisons bâties sur pilotis au-dessus de la rivière (Goubet: 394 ) : toutes sortes de constructions humaines paraissent soudain bien frêles lorsque se déchaînent les puissances de la nature. En 1801, 1802, 1807, 1817 et 1836 la magnitude des ravages est notable et le niveau d'eau particulièrement impressionnant (Goubet: 398), même si on reste loin des maxima atteints en 1910. Cependant, dans les premières décennies du XIXe siècle la fréquence des crues signalées et rapportées rejoint des sommets statistiques inégalés (Lescure). C'est d'ailleurs à partir de cette période que l'État entreprend de bâtir, en amont de la Capitale, des ouvrages d'ingénierie destinés à réguler le débit d'eau et atténuer le fléau; ces divers dispositifs de contention 
hydrologique sont essentiellement des barrages.

29. Ce n'est que depuis la fin de l'Ancien Régime qu'on connaît les dangers liés aux eaux stagnantes.

30. Le jardin des Tuileries en particulier et les bords de Seine en général sont des foyers de prostitution et de pédérastie bien connus des policiers tout le long du siècle (Sibalis: 16-18).

31. Il faut savoir qu'à l'époque les quais de Seine sont rarement aménagés à la verticale comme ils le sont aujourd'hui; la plupart des ports, établis en pleine ville, se composent de quais de bois posés sur des grèves boueuses, jonchées de détritus; les bateaux s'échouent là pour déposer leurs marchandises; des îlots de sable se forment et se reforment de saison en saison, çà et là, au gré des courants, formant des marécages en maints endroits, retenant les déchets charriés par l'eau; des épaves d'anciennes barques pourrissent, éventrées, parmi les morceaux d'écorce, les branches cassées, tous les résidus de l'industrie forestière qui achemine sa marchandise à Paris par flottage (Millard: 43-45).

32. On repensera au cri poussé par cette vieille dame en haillons croisée par Raphaël de Valentin sur le pont Royal au début de $L a$ Peau de Chagrin : «Est-elle sale et froide, la Seine! » (Balzac, 1979 [1831]: 65.)

33. Au Moyen-Âge, saint Marcel incarne «mieux et plus » que tout autre « l'Église parisienne, le siège épiscopal parisien, la communauté chrétienne parisienne », note Jacques Le Goff (265). La primauté du culte de Marcel et de son dragon perdure à Paris jusqu'à ce que les nouvelles valeurs portées par la Révolution l'éclipsent au profit de sainte Geneviève et saint Denis (Le Goff: 239).

34. Deux des trois cas concernent explicitement l'eau de la Seine (Le Goff: 237); et puisque le troisième s'est produit à Paris tout autant que les autres, force est de supposer qu'il s'agit encore d'eau de Seine, puisée à même le fleuve, à moins qu'elle vienne de quelque proche affluent, comme la Bièvre, ce qui revient à peu près au même en l'absence de toute indication expresse.

35. Rappelons avec Jacques Le Goff (238) que les reliques de saint Marcel furent transportées à Notre-Dame de Paris entre le $X^{e}$ et le XII ${ }^{e}$ siècle. Aujourd'hui, elles sont conservées à l'église Saint-Marcel de Paris, dans le treizième arrondissement, près du quartier des Gobelins, nommé en l'honneur de la célèbre teinturerie s'étant établie là, sur les berges de la Bièvre, au début du $\mathrm{XVII}$ siècle.

36. Si les premières tentatives de composer une poésie en prose, notamment celles d'Alphonse Rabbe et d'Aloysius Bertrand, sont à peu près contemporaines du poème de Vigny, la versification reste encore l'unique véritable véhicule poétique de l'époque. II faut attendre la seconde moitié du siècle pour que Baudelaire s'autorise à hacher le serpent poétique en segments inégaux dans $L e$ Spleen de Paris (1869) et la fin du siècle pour que Stéphane Mallarmé fasse éclater le vers avec son Coup de dés (1897).

\section{Bibliographie}

Baldensperger, Fernand. 1950. «Notes et additions aux poésies », dans Alfred Vigny (DE) (dir.), CEuvres complètes, texte présenté et commenté par Fernand Baldensperger. Paris : Gallimard, « Bibliothèque de la Pléiade », t. 1, p. 175-264.

BALzAC (DE), Honoré. 1979 [1831]. «La Peau de chagrin », dans La Comédie humaine, sous la direction de Pierre-Georges Castex. Paris : Gallimard, « Bibliothèque de la Pléiade », t. 10, p. 48-294.

BALZAC (DE), Honoré. 1976 [1834]. «Le Père Goriot », dans La Comédie humaine, sous la direction de PierreGeorges Castex. Paris : Gallimard, « Bibliothèque de la Pléiade », t. 3, p. 37-290.

BALZAC (DE), Honoré. 1979 [1837]. « Le Chef d'œuvre inconnu », dans La Comédie humaine, sous la direction de Pierre-Georges Castex. Paris : Gallimard, « Bibliothèque de la Pléiade », t. 10, p. 413-438.

Baudelaire, Charles. 1999 [1845]. «Salon de 1845 », dans Écrits sur l'art, texte établi, présenté et annoté par

Francis Moulinat. Paris : Librairie générale française, « Le Livre de Poche. Classique », p. 49-121.

Baudelaire, Charles. 1999 [1846]. «Salon de 1846 », dans Écrits sur l'art, texte établi, présenté et annoté par

Francis Moulinat. Paris : Librairie générale française, « Le Livre de Poche. Classique », p. 135-242.

BÉnichou, Paul. 1988. Les Mages romantiques. Paris : Gallimard, « Bibliothèque des Idées », 553 p. 
Benjamin, Walter. 2006. Paris, capitale du XIXe siècle. Le Livre des passages. Paris : Cerf, "Passages », $976 \mathrm{p}$.

Bertrand, Jean-Pierre et Pascal Durand. 2006. La Modernité romantique. De Lamartine à Nerval Paris; Bruxelles : Impressions nouvelles, $236 \mathrm{p}$.

BonaPARTE, Napoléon. 1861 [1800]. «Allocution aux curés de la ville de Milan. 5 juin 1800 », dans Correspondance de Napoléon 1er. Paris : Plon; Dumaine, t. 6, p. 338-340.

Chaumont, Bérangère. 2016. «Effets de nuit dans la poésie de Vigny», dans Lise SABourin et Sylvain LedDA (dir.), Poétique de Vigny. Paris : Champion, « Romantisme et modernités », p. 66-84.

Citron, Pierre. 1961. La Poésie de Paris dans la littérature française de Rousseau à Baudelaire Paris : Minuit, t. 1, 437 p.

Drissa, Mohamed Ali. 1979. Vigny et le symbole. Tunis : Publications de l'Université de Tunis, «Philosophie et littérature », $309 \mathrm{p}$.

Flaubert, Gustave. 1979 [1913]. «Dictionnaire des idées reçues », dans Bouvard et Pécuchet, édition présentée et établie par Claudine Gothot-Mersch. Paris : Gallimard, « Folio classique », p. 486-555.

Flottes, Alfred. 2012 [1927]. La Pensée politique et sociale d'Alfred de Vigny. Genève : Slatkine, $360 \mathrm{p}$.

GAUCHER, Maxime. 1873. «Causerie littéraire ». Revue bleue. Politique et littéraire, 2e série, vol. 2, no 40, 5 avril, p. 969-972.

GOuBET, André. 1981. «Les crues dans le bassin de la Seine du XVIle siècle au début du XIXe siècle». La Houille blanche, no 6, p. 393-404. <http://www.shf-lhb.org/articles/lhb/pdf/1981/04/lhb1981040.pdf>.

GRAS, Alain. 2007. «Du lavoir à la machine à laver. Sommes-nous dépendants des systèmes techniques? », dans Hana Aubry (dir.), Imaginaires de l'eau, imaginaire du monde. Dix regards sur l'eau et sa symbolique dans les sociétés humaines, préface de Larbi Bouguerra, postface de Jacques Perreux. Paris : La Dispute, «Tout autour de l'eau », p. 83-106.

GuILleRmE, André. 2007. «De l'eau sacralisée à l'eau canalisée. L'histoire de l'eau dans les villes du Bassin parisien », dans Hana AuBry (dir.), Imaginaires de l'eau, imaginaire du monde. Dix regards sur l'eau et sa symbolique dans les sociétés humaines, préface de Larbi Bouguerra, postface de Jacques Perreux. Paris : La Dispute, « Tout autour de l'eau », p. 33-52.

JARRY, André. 1973. « Notices et notes », dans Alfred VIGNY (DE) (dir.), Poèmes antiques et modernes. Les Destinées, préface de Marcel Arland, texte établi et annoté par André Jarry. Paris : Gallimard, "Poésie », p. 271-309.

JARRY, André. 1998. Alfred de Vigny. Étapes et sens du geste littéraire, lecture psychanalytique Genève :

Droz, « Histoire des idées et critique littéraire », t. 1, 537 p.

KALIFA, Dominique. 2013. Les Bas-fonds. Histoire d'un imaginaire. Paris : Seuil, «L'univers historique », $394 \mathrm{p}$. 
LAFORGUE, Pierre. 2003. «Machinisme et industrialisme, ou romantisme, modernité et mélancolie.

Quelques jalons (1840-1870) ». Revue d'histoire littéraire de la France, vol. 103, no 1, p. 63-92.

Lambeau, Lucien. 1906. «À propos de l'échafaudage de la tour Saint-Jacques-la-Boucherie. Quelques

pages d'histoire moderne d'un vieux monument ». Procès-verbaux. Commission municipale du Vieux Paris, no 8, séance du samedi 10 novembre, p. 267-288.

$<$ http://gallica.bnf.fr/ark:/12148/bpt6k58218061/f44.item.zoom>.

LE GofF, Jacques. 1991. «Culture ecclésiastique et culture folklorique au Moyen-Âge. Saint Marcel de

Paris et le dragon », dans Pour un autre Moyen-Âge. Temps, travail et culture en Occident Paris :

Gallimard, «Tel », p. 236-279.

Lescure, Séverine, Gilles Arnaud-Fassetta et Stéphane Corder. 2011. «Sur quelques modifications hydromorphologiques dans le Val de Seine (Bassin parisien, France) depuis 1830. Quelle part accorder aux facteurs hydrologiques et anthropiques? ». EchoGéo, no 18, septembre-décembre. $<$ http://journals.openedition.org/echogeo/12658>.

Millard, Jean. 1994. Paris, histoire d'un port. Du Port de Paris au Port autonome de Paris préface de Bernard Chevenez. Paris : L'Harmattan, 175 p.

Petroni, Liano. 1990. «Un exemple peu courant de la description d'une ville dans le romantisme français. Le Paris de "Stello" ». Cahiers de l'Association internationale des études françaises, no 42, p. 19-29.

Sibalis, Michael D. 1999. «Paris », dans David Higgs (dir.), Queer sites. Gay Urban Histories since 1600 Londres : Routledge, p. 10-37.

Tervarent (DE), Guy. 1997 [1958]. Attributs et symboles dans l'art profane. Dictionnaire d'un langage perdu (1450-1600). Genève : Droz, «Titre courant », 536 p.

TUdesQ, André-Jean. 1958. «Les listes électorales de la Monarchie censitaire ». Annales. Économie, Sociétés, Civilisations, vol. 13, no 2, p. 277-288. <http://www.persee.fr/doc/ahess 0395$\underline{2649} 1958$ num $1322736>$.

VIGNY (DE), Alfred. 1950 [1859]. «Paris », dans CEuvres complètes. Poèmes antiques et modernes texte présenté et commenté par Fernand Baldensperger. Paris : Gallimard, «Bibliothèque de la Pléiade », t. 1, p. $109-115$.

VIGNY (DE), Alfred. 1867. Journal d'un poëte, recueilli et publié sur des notes intimes d'Alfred de Vigny par Louis Ratisbonne. Paris : Michel Lévy Frères, 307 p. 